\title{
On the spectral properties of the Schrodinger operator with a periodic PT-symmetric potential
}

\author{
O. A. Veliev \\ Depart. of Math., Dogus University, \\ Acıbadem, 34722, Kadiköy, Istanbul, Turkey. \\ e-mail: oveliev@dogus.edu.tr
}

\begin{abstract}
In this paper we investigate the spectrum and spectrality of the one-dimensional Schrodinger operator with a periodic PT-symmetric complex-valued potential.

Key Words: Schrodinger operator, PT-symmetric periodic potential, Real spectrum.

AMS Mathematics Subject Classification: 34L05, 34L20.
\end{abstract}

\section{Introduction and Preliminary Facts}

In this paper we investigate the one dimensional Schrödinger operator $L(q)$ generated in $L_{2}(-\infty, \infty)$ by the differential expression

$$
-y^{\prime \prime}(x)+q(x) y(x)
$$

where $q$ is complex-valued, locally integrable, periodic and PT-symmetric. Without loss of generality, we assume that the period of $q$ is 1 and the integral of $q$ over $[0,1]$ is zero. Thus

$$
q \in L_{1}[0,1], \quad \int_{0}^{1} q(x) d x=0, q(x+1)=q(x), \overline{q(-x)}=q(x) \text { (a.e.). }
$$

A basic mathematical question of PT-symmetric quantum mechanics concerns the reality of the spectrum of the considered Hamiltonian (see $[2,15$ and references of them]). In the first papers $[1,3,5,6,10]$ about the PT-symmetric periodic potential, the appearance and disappearance of real energy bands for some complex-valued PT-symmetric periodic potentials under perturbations have been reported. Shin [17] showed that the appearance and disappearance of such real energy bands imply the existence of nonreal band spectra. He involved some condition on the Hill discriminant to show the existence of nonreal curves in the spectrum. Caliceti and Graffi [4] found explicit condition on the Fourier coefficient of the potential providing the nonreal spectra for small potentials. Besides, they proved that if all gaps of the spectrum of the Hill operator $L(q)$ with distributional potential $q$ are open and the width of the $n$-th gap does not vanish as $n \rightarrow \infty$, then the spectrum of $L(q)+g W$, where $W$ is a bounded periodic function and $g$ is a small number, is real. This result can not be used for the locally integrable periodic potentials (2), since the width of the $n$-th gap vanishes as $n \rightarrow \infty$.

In this paper, we first consider the general spectral property of the spectrum of $L(q)$ under conditions (2) and prove that the main part of its spectrum is real and contains the large part of $[0, \infty)$. Using this we find necessary and sufficient condition on the potential 
for finiteness of the number of the nonreal arcs in the spectrum of $L(q)$. Besides we find necessary and sufficient conditions for the equality of the spectrum of $L(q)$ to the half line. Moreover, we consider the connections between spectrality of $L(q)$ and the reality of its spectrum for some class of PT-symmetric periodic potentials. Finally, we find explicit conditions on the potential $q$ for which the number of gaps in the real part of the spectrum of $L(q)$ is finite.

Now let us list the well-known results, as Summary 1-Sammary 7, about $L(q)$ which will be essentially used in this paper. Note that we formulate the well-known results from the books $[7,19]$ and the papers $[11,13,14,16,17]$ in the suitable form for this paper and by using the unique notation, since the different notations were used in those references.

Summary 1 The spectrum $\sigma(L)$ of the operator $L$ is the union of the spectra $\sigma\left(L_{t}\right)$ of the operators $L_{t}$ for $t \in(-\pi, \pi]$ generated in $L_{2}[0,1]$ by (1) and the boundary conditions

$$
y(1)=e^{i t} y(0), y^{\prime}(1)=e^{i t} y^{\prime}(0) .
$$

Summary 2 The eigenvalues of $L_{t}$ are the roots of the characteristic equation

$$
F(\lambda)=2 \cos t,
$$

where $F(\lambda):=\varphi^{\prime}(1, \lambda)+\theta(1, \lambda)$ is the Hill discriminant, $\theta$ and $\varphi$ are the solutions of

$$
-y^{\prime \prime}(x)+q(x) y(x)=\lambda y(x)
$$

satisfying the initial conditions $\theta(0, \lambda)=\varphi^{\prime}(0, \lambda)=1, \theta^{\prime}(0, \lambda)=\varphi(0, \lambda)=0$.

The following 2 summaries immediately follows from summaries 1 and 2 .

Summary $3 \sigma\left(L_{-t}\right)=\sigma\left(L_{t}\right)=\sigma\left(L_{t+2 \pi}\right)$ and $\lambda \in \sigma\left(L_{t}\right)$ if and only if there exist a solution $\Psi(x, \lambda)$ of (5) satisfying $\Psi(x+1, \lambda)=e^{i t} \Psi(x, \lambda)$ for all $x$.

Summary $4 \lambda \in \sigma(L)$ if and only if $F(\lambda) \in[-2,2]$.

The following summary was proved in $[16,20]$

Summary 5 The spectrum $\sigma(L)$ consist of analytic arcs whose endpoints are the eigenvalues of $L_{t}$ for $t=0, \pi$ and the multiple eigenvalues of $L_{t}$ for $t \in(0, \pi)$. Moreover $\sigma(L)$ does not contain the closed curves, that is, the resolvent set $\mathbb{C} \backslash \sigma(L)$ is connected.

Let (2) holds. Then using Summary 3 one can readily see that the function $\Phi(x, \lambda):=$ $\overline{\Psi(-x, \lambda)}$ satisfies the equation $-y^{\prime \prime}(x)+q(x) y(x)=\bar{\lambda} y(x)$ and the equality

$$
\Phi(x+1, \lambda)=\overline{\Psi(-x-1), \lambda)}=\overline{e^{-i t} \Psi(-x, \lambda)}=e^{i t} \Phi(x, \lambda) .
$$

It means that $\bar{\lambda} \in \sigma\left(L_{t}\right)$. Hence we have

Summary 6 If (2)holds then the following implications hold

$$
\lambda \in \sigma\left(L_{t}\right) \Longrightarrow \bar{\lambda} \in \sigma\left(L_{t}\right), \lambda \in \sigma(L) \Longrightarrow \bar{\lambda} \in \sigma(L) .
$$

The first and second implications were proved in [11] and [17] respectively.

The following summary is proved in [17] (see Theorem 3 and Corollary 4).

Summary 7 Suppose (2) holds. Then $F(\lambda)$ is real for all real $\lambda$, where $F(\lambda)$ is defined in (4). If $F(\lambda) \in(-2,2)$ and $F^{\prime}(\lambda)=0$ or $F(\lambda)= \pm 2, F^{\prime}(\lambda)=0$ and $F^{\prime \prime}(\lambda)=0$ then $\sigma(L(q))$ contains nonreal number in any neighborhood of $\lambda$.

In Section 2 we use these summaries and the uniform asymptotic formulas for the eigenvalues of $L_{t}(q)$ obtained in [22] and [23] and formulated as Summary 8 and Summary 9 in order to describe the spectrum of the operator $L(q)$ with the potential (2). In the last section using the results of Section 2 and the papers $[23,27]$ we consider in detail the shape of the spectrum and the asymptotic connection of reality of $\sigma(L)$ and spectrality of $L$. 


\section{On the Spectrum of $L(q)$}

In the case $q=0$ the eigenvalues and eigenfunctions of $L_{t}(q)$ are $(2 \pi n+t)^{2}$ and $e^{i(2 \pi n+t) x}$ for $n \in \mathbb{Z}$ respectively. In [27] we proved that the eigenvalues of $L_{t}$ can be numbered (counting the multiplicity) by elements of $\mathbb{Z}$ such that, for each $n$ the function $\lambda_{n}(t)$ is a piecewise differentiable on $[0, \pi]$ and $\lambda_{n}(-t)=\lambda_{n}(t)$ (see Summary 3). Thus

$$
\sigma(L(q))=\bigcup_{n \in \mathbb{Z}} \Gamma_{n}
$$

where $\Gamma_{n}=\left\{\lambda_{n}(t): t \in[0, \pi]\right\}$ (see (16) of [27]). In future we call $\Gamma_{n}$ as band of $\sigma(L(q)$ ). Moreover, it follows from the construction of $\Gamma_{n}$ that

$$
\Gamma_{n} \cap \sigma\left(L_{t}(q)\right)=\left\{\lambda_{n}(t)\right\}, \quad\left|\Gamma_{n}\right|<\infty, \lim _{n \rightarrow \infty} d\left(\Gamma_{n}, 0\right)=\infty,
$$

where $\left|\Gamma_{n}\right|$ is the length of $\Gamma_{n}$ and $d\left(\Gamma_{n}, 0\right)$ is the distance from $\Gamma_{n}$ to origin. Besides one can readily see the following properties of the bands $\Gamma_{n}$.

Proposition 1 (a) $\Gamma_{n}$ is a single open curve with the end points $\lambda_{n}(0)$ and $\lambda_{n}(\pi)$.

(b) Two bands $\Gamma_{n}$ and $\Gamma_{m}$ may have at most one common point.

Proof. (a) It follows from (3) and (4) that $\lambda_{n}\left(t_{1}\right) \neq \lambda_{n}\left(t_{2}\right)$ for $0 \leq t_{1}<t_{2} \leq \pi$. Therefore $\Gamma_{n}$ is a single open curve and hence has two end points. Since $\lambda_{n}:[0, \pi] \rightarrow \Gamma_{n}$ is a piecewise differentiable functions it maps the ends of $[0, \pi]$ to the ends of $\Gamma_{n}$.

(b) The inequality $\lambda_{n}\left(t_{1}\right) \neq \lambda_{m}\left(t_{2}\right)$ for $t_{1} \neq t_{2}$ implies that the bands $\Gamma_{n}$ and $\Gamma_{m}$ has a common point if and only if there exist $t \in[0, \pi]$ such that $\lambda_{n}(t)=\lambda_{m}(t)$. It means that $\lambda_{n}(t)$ is a multiple eigenvalue of $L_{t}$ and hence is the roots of $F^{\prime}(\lambda)=0$, where $F(\lambda)$ is the Hill discriminant defined in Summary 2. Since $F(\lambda)$ is a nonzero entire function, the set of zeros of $F^{\prime}(\lambda)$ is a discrete set and has no finite limit points. Therefore, by the inequality in (7), two bands can have only finite number common points. If the common points of $\Gamma_{n}$ and $\Gamma_{m}$ are more than one, then there exist $t_{1}$ and $t_{2}$ such that $0 \leq t_{1}<t_{2} \leq \pi$ and

$$
\lambda_{n}\left(t_{1}\right)=\lambda_{m}\left(t_{1}\right), \lambda_{n}\left(t_{2}\right)=\lambda_{m}\left(t_{2}\right), \lambda_{n}\left(t_{1}\right) \neq \lambda_{n}\left(t_{2}\right), \lambda_{n}(t) \neq \lambda_{m}(\tau)
$$

for all $t \in\left(t_{1}, t_{2}\right)$ and $\tau \in\left(t_{1}, t_{2}\right)$. However, it implies that

$$
\left\{\lambda_{n}(t): t \in\left[t_{1}, t_{2}\right]\right\} \cup\left\{\lambda_{m}(t): t \in\left[t_{1}, t_{2}\right]\right\}
$$

is a closed curve which contradicts Summary 5.

Remark 1 Note that if $\lambda_{n}(t)$ for $t \in(0, \pi)$ and $t=0, \pi$ is a multiple eigenvalue of $L_{t}(q)$ of multiplicity $p$, then $p$ bands of the spectrum have common interior and end point $\lambda_{n}(t)$ respectively. In particular, $\lambda_{n}(t)$ is a simple eigenvalue if and only if it belong only to one band $\Gamma_{n}$.

These arguments with summaries 5 and 6 yield the following

Theorem 1 Suppose that (2) holds.

(a) If $\lambda_{n}\left(t_{1}\right)$ and $\lambda_{n}\left(t_{2}\right)$ are real numbers, where $0 \leq t_{1}<t_{2} \leq \pi$ then $\gamma:=\left\{\lambda_{n}(t): t \in\left[t_{1}, t_{2}\right]\right\}$ is an interval of the real line.

(b) If the eigenvalues $\lambda_{n}(0)$ and $\lambda_{n}(\pi)$ are real numbers then $\lambda_{n}(t)$ are real eigenvalues of $L_{t}(q)$ for all $t \in(0, \pi)$, that is, $\Gamma_{n}$ is either $\left[\lambda_{n}(0), \lambda_{n}(\pi)\right]$ or $\left[\lambda_{n}(\pi), \lambda_{n}(0)\right]$.

(c) The spectrum of $L(q)$ is completely real if and only if all eigenvalues of $L_{0}(q)$ and $L_{\pi}(q)$ are real, that is, the roots of $(F(\lambda))^{2}=4$ are the real numbers. 
Proof. (a) If $\gamma$ is not an interval of the real line, then, by Summary $6, \sigma(L)$ contains a closed curve $\gamma \cup \widetilde{\gamma}$, where $\widetilde{\gamma}=\{\lambda \in \mathbb{C}: \bar{\lambda} \in \gamma\}$, which contradicts Summary 5.

(b) Since $\lambda_{n}(0)$ and $\lambda_{n}(\pi)$ are the end points of $\Gamma_{n},(b)$ follows from $(a)$.

(c) If the spectrum of $L(q)$ is real, then by Summary 1 the eigenvalues of $L_{0}(q)$ and $L_{\pi}(q)$ are real. Now suppose that all eigenvalues of $L_{0}(q)$ and $L_{\pi}(q)$ are real. It means that the eigenvalues $\lambda_{n}(0)$ and $\lambda_{n}(\pi)$ are real numbers for all $n$. Then, by $(b), \Gamma_{n}$ for all $n$ and hence, by $(6), \sigma(L)$ is completely real.

Now to consider, the reality of the spectrum in detail, we investigate the points in which the spectrum ceases to be real. These points are crucial and can be defined as follows.

Definition $1 A$ real number $\lambda \in \sigma(L)$ is said to be a left (right) complexation point of the spectrum if there exists $\varepsilon>0$ such that $[\lambda, \lambda+\varepsilon] \subset \sigma(L)([\lambda-\varepsilon, \lambda] \subset \sigma(L))$ and $\sigma(L)$ contains a nonreal number in any neighborhood of $\lambda$. Both left and right complexation points are called complexation points.

Theorem 2 Suppose that (2) holds. If $\lambda_{n}(t)$ is a complexation point, then it is a multiple eigenvalue. Moreover, the multiplicity of $\lambda_{n}(t)$ is greater than 2 if $t=0, \pi$.

Proof. Let $\lambda_{n}(t)$ be a complexation point. If $\lambda_{n}(t)$ is a simple eigenvalue, then by Remark 1, $\lambda_{n}(t)$ belong to $\Gamma_{n}$ and does not belong to $\Gamma_{m}$ for $m \neq n$. Moreover, there exists a neighborhood $U:=\left\{\lambda \in \mathbb{C}:\left|\lambda-\lambda_{n}(t)\right|<\delta\right\}$ of $\lambda_{n}(t)$ such that $U \cap \Gamma_{m}=\varnothing$ for all $m \neq n$. Then by Definition 1 and Summary 6 there exist a nonreal number $\lambda \in U$ and $t_{0} \in(0, \pi)$ such that $\lambda \in\left(\Gamma_{n} \cap \sigma\left(L_{t_{0}}(q)\right)\right)$ and $\bar{\lambda} \in\left(\Gamma_{n} \cap \sigma\left(L_{t_{0}}(q)\right)\right)$ which contradicts (7).

Now suppose that $\lambda_{n}(0)$ is a complexation point and multiple eigenvalue of multiplicity two. Then, by Remark 1 , there exists $m \neq n$ such that $\lambda_{m}(0)=\lambda_{n}(0)$ and $\lambda_{k}(0) \neq \lambda_{n}(0)$ for all $k \neq n, m$. Therefore using Definition 1 and taking into account the continuity of $\lambda_{k}(t)$ for all $k \in \mathbb{Z}$, we see that there exist $\varepsilon>0$ and $\delta>0$ such that at least one of the sets $\left\{\lambda_{n}(t): t \in[0, \varepsilon]\right\}$ and $\left\{\lambda_{m}(t): t \in[0, \varepsilon]\right\}$ is the interval of the real line and

$$
\left\{\lambda \in \mathbb{C}:\left|\lambda-\lambda_{n}(0)\right|<\delta\right\} \cap\left\{\lambda_{k}(t): t \in[0, \varepsilon]\right\}=\varnothing, \forall k \neq n, m .
$$

Suppose, without loss of generality, that $\left\{\lambda_{n}(t): t \in[0, \varepsilon]\right\} \subset \mathbb{R}$. Then by (8) and Definition 1 there exist $t \in[0, \varepsilon]$ and $\delta>0$ such that $\lambda_{m}(t) \in\left\{\lambda \in \mathbb{C}:\left|\lambda-\lambda_{n}(0)\right|<\delta\right\} \backslash \mathbb{R}$. These arguments with (7), (8) and Summary 6 imply that $\overline{\lambda_{m}(t)}$ belong to $\sigma(L(q))$ and does not belong to $\Gamma_{k}$ for any $k \in \mathbb{Z}$. It contradicts (6). In the same way we consider $\lambda_{n}(\pi)$

Now we consider the large eigenvalues by using the uniform asymptotic formulas for the Bloch eigenvalues obtained in $[22,23]$ and then combining it with the above results we will describe the spectrum lying outside of some disk. In [22] (see Theorem 2) we proved that the large eigenvalues of the operators $L_{t}(q)$ for $t \neq 0, \pi$ consist of the sequence $\left\{\lambda_{n}(t):|n| \gg 1\right\}$ satisfying

$$
\lambda_{n}(t)=(2 \pi n+t)^{2}+O\left(n^{-1} \ln |n|\right)
$$

as $n \rightarrow \infty$ and the formula (9) is uniform with respect to $t$ in $[h, \pi-h]$, where $h$ is a fixed number and, without loss of generality, it is assumed that the integral of $q$ over $[0,1]$ is 0 . In future we assume that $h \in(0,1 / 15 \pi)$. The following summary follows from (9).

Summary 8 For any fixed $h$, there exists an integer $N(h)$ and positive constant $M(h)$ such that for $|n|>N(h)$ and $t \in[h, \pi-h]$ there exists unique, counting multiplicity, eigenvalue $\lambda_{n}(t)$ satisfying

$$
\left|\lambda_{n}(t)-(2 \pi n+t)^{2}\right| \leq M(h) n^{-1} \ln n, \quad\left|\lambda_{n}(t)-\lambda_{k}(t)\right|>2 \pi^{2} n, \quad \forall k \neq n .
$$

The eigenvalue $\lambda_{n}(t)$ is simple for all $|n|>N(h)$ and $t \in[h, \pi-h]$. 
Moreover, as it was shown in [23] (see (20) and Remark 2.11of [23]) and [27] (see page 60 of $[27]) N(h)$ can be chosen so that the following holds.

Summary 9 For $t \in[0, h]$ and $n>N(h)$ there exist two eigenvalues, counting multiplicity, denoted by $\lambda_{n}(t)$ and $\lambda_{-n}(t)$ and satisfying

$$
\left|\lambda_{ \pm n}(t)-(2 \pi n+t)^{2}\right| \leq 15 \pi n h<n, \quad\left|\lambda_{ \pm n}(t)-\lambda_{k}(t)\right|>2 \pi^{2} n, \forall k \neq \pm n .
$$

Similarly, for $t \in[\pi-h, \pi]$ and $n>N(h)$ there exist two eigenvalues, counting multiplicity, denoted by $\lambda_{n}(t)$ and $\lambda_{-n-1}(t)$ such that

$$
\begin{aligned}
\left|\lambda_{n}(t)-(2 \pi n+t)^{2}\right| & \leq 15 \pi n h<n, \quad\left|\lambda_{-n-1}(t)-(2 \pi n+t)^{2}\right| \leq 15 \pi n h<n, \\
\left|\lambda_{n}(t)-\lambda_{k}(t)\right| & >2 \pi^{2} n, \quad\left|\lambda_{-n-1}(t)-\lambda_{k}(t)\right|>2 \pi^{2} n, \quad \forall k \neq n,-(n+1) .
\end{aligned}
$$

In [27] we proved that the eigenvalues of $L_{t}$ can be numbered (counting the multiplicity) by elements of $\mathbb{Z}$ such that, for each $n$ the function $\lambda_{n}(t)$ is continuous on $[0, \pi]$ and for $|n|>N(h)$ the inequalities (10)-(12) hold. Moreover, the bands $\Gamma_{n}$ of the spectrum defined in (6) is constructed due to this numerations. Therefore inequalities (11) and (12) and Proposition 1 yield the following.

Proposition 2 Suppose that $t \in([0, h] \cup[\pi-h, \pi])$ and $|n|>N(h)$. Then

(a) The multiplicity of the eigenvalues $\lambda_{n}(t)$ is not greater than 2 .

(b) If $\lambda_{n}(t)$ for $t \in[0, h]$ and $|n|>N(h)$ is the double eigenvalue of $L_{t}$, then $\lambda_{n}(t)=$ $\lambda_{-n}(t)$ and hence the bands $\Gamma_{n}$ and $\Gamma_{-n}$ have the common point $\lambda_{n}(t)$. Similarly, if $\lambda_{n}(t)$ for $t \in[\pi-h, \pi]$ and $|n|>N(h)$ is the double eigenvalue of $L_{t}$, then $\lambda_{n}(t)=\lambda_{-n-1}(t)$ and hence the bands $\Gamma_{n}$ and $\Gamma_{-n-1}$ have the common point $\lambda_{n}(t)$.

(c) If $n$ is a positive (negative) number, then the left and right ends of $\Gamma_{n}$ are $\lambda_{n}(0)$ $\left(\lambda_{n}(\pi)\right)$ and $\lambda_{n}(\pi)\left(\lambda_{n}(0)\right)$ respectively.

Now using the above arguments we describe the shapes of $\Gamma_{n}$ for $|n|>N(h)$.

Theorem 3 Suppose that (2) holds and $|n|>N(h)$.

(a) $\lambda_{n}(t)$ for $t \in[h, \pi-h]$ are real and simple eigenvalues.

(b) For $t \in([0, h] \cup[\pi-h, \pi])$ the double eigenvalues $\lambda_{n}(t)$ are the real numbers.

Proof. (a) By Summary $8, \lambda_{n}(t)$ for $t \in[h, \pi-h]$ are the simple eigenvalues. If $\lambda_{n}(t) \notin \mathbb{R}$ for some $t \in[h, \pi-h]$, then $\overline{\lambda_{n}(t)} \neq \lambda_{n}(t)$ and by Summary 6 both are eigenvalues of $L_{t}(q)$ satisfying (10), which contradicts Summary 8.

(b) If the double eigenvalue $\lambda_{n}(t)$ for $t \in[0, h]$ is nonreal then arguing as in the proof of (a) we conclude that there exist four eigenvalues (counting the multiplicity) satisfying (11) which contradicts Summary 9. In the same way the case $t \in[\pi-h, \pi]$ can be considered

Thus the part $\left\{\lambda_{n}(t): t \in[h, \pi-h]\right\}$ of $\Gamma_{n}$ is the interval of the real line. The following theorem shows that, in fact, very large part of $\Gamma_{n}$ consists of the interval of the real line.

Theorem 4 Suppose that (2) holds and $|n|>N(h)$.

(a) There may exists at most one number $\varepsilon_{n}$ and $\pi-\delta_{n}$ in $[0, h)$ and $(\pi-h, \pi]$ respectively, such that $\lambda_{n}\left(\varepsilon_{n}\right)$ and $\lambda_{n}\left(\pi-\delta_{n}\right)$ are double eigenvalues. Then

$$
\lambda_{n}\left(\varepsilon_{n}\right)=\lambda_{-n}\left(\varepsilon_{n}\right) \in \mathbb{R}, \lambda_{n}\left(\pi-\delta_{n}\right)=\lambda_{-n-1}\left(\pi-\delta_{n}\right) \in \mathbb{R}, \forall n>N(h) .
$$

(b) The eigenvalues $\lambda_{n}(t)$ for $t \neq[0, \pi] \backslash\left\{\varepsilon_{n}, \pi-\delta_{n}\right\}$ are simple and are not complexation points. Moreover $\varepsilon_{n} \rightarrow 0$ and $\delta_{n} \rightarrow 0$ as $n \rightarrow \infty$.

(c) The double eigenvalues $\lambda_{n}\left(\varepsilon_{n}\right)$ and $\lambda_{n}\left(\pi-\delta_{n}\right)$ are complexation points if and only if $\varepsilon_{n} \neq 0$ and $\delta_{n} \neq 0$ respectively. 
(d) If $\lambda_{n}\left(\varepsilon_{n}\right)$ and $\lambda_{n}\left(\pi-\delta_{n}\right)$ are complexation points, then the part $\left\{\lambda_{n}(t): t \in\left[\varepsilon_{n}, \pi-\delta_{n}\right]\right\}$ of $\Gamma_{n}$ is an interval of the real line and is the real part $\operatorname{Re}\left(\Gamma_{n}\right)$ of $\Gamma_{n}$ and the other parts $\gamma\left(0, \varepsilon_{n}\right):=\left\{\lambda_{n}(t): t \in\left[0, \varepsilon_{n}\right)\right\}$ and $\gamma\left(\pi, \delta_{n}\right):=\left\{\lambda_{n}(t): t \in\left(\pi-\delta_{n}, \pi\right]\right\}$ are not empty set and are the pure nonreal parts of $\Gamma_{n}$, that is, belong to $\mathbb{C} \backslash \mathbb{R}$.

Proof. (a) Suppose to the contrary that there exist two numbers $t_{1}$ and $t_{2}$ such that both $\lambda_{n}\left(t_{1}\right)$ and $\lambda_{n}\left(t_{2}\right)$ are the double eigenvalues, where $0 \leq t_{1}<t_{2}<h$. Then by Proposition $2(b)$ the bands $\Gamma_{n}$ and $\Gamma_{-n}$ have two common points $\lambda_{n}\left(t_{1}\right)$ and $\lambda_{n}\left(t_{2}\right)$ which contradicts Proposition $1(b)$. The case $(\pi-h, \pi]$ can be considered in the same way. The equalities and inclusions in (13) follows from Proposition 2(b) and Theorem 3(b) respectively.

(b) The simplicity of eigenvalues $\lambda_{n}(t)$ for $t \neq[0, \pi] \backslash\left\{\varepsilon_{n}, \pi-\delta_{n}\right\}$ follows from Summary 8 and definitions of $\varepsilon_{n}$ and $\pi-\delta_{n}$. Then by Theorem 2 these eigenvalues are not complexation points. The relations $\varepsilon_{n} \rightarrow 0$ and $\delta_{n} \rightarrow 0$ as $n \rightarrow \infty$ are well-known. It readily follows from Summary 8 and from the asymptotic formulas

$$
F(\lambda)=2 \cos \sqrt{\lambda}+O(1 / \sqrt{\lambda})
$$

as $\lambda \in \mathbb{R}$ and $\lambda \rightarrow \infty$ (see [12,Chap.1, Sec.3] and [22, page 78]).

(c) Theorem 2 implies that $\lambda_{n}(t)$ for $t=0, \pi$ are not complexation points since by Proposition 2(a) their multiplicities is not greater than 2. Therefore it follows from $(b)$ that if $\lambda_{n}(t)$ is a complexation point, then either $t=\varepsilon_{n} \neq 0$ or $t=\pi-\delta_{n} \neq \pi$. Consider $\lambda_{n}(t)$ for $t=\varepsilon_{n}$ and $\varepsilon_{n} \neq 0$. By Theorem $3, \lambda_{n}\left(\varepsilon_{n}\right)$ and $\lambda_{n}(h)$ are real numbers. Therefore by Theorem 1 the set $\left\{\lambda_{n}(t): t \in\left[\varepsilon_{n}, h\right]\right\} \subset \Gamma_{n}$ is an interval of the real line. Since $\varepsilon_{n} \neq 0, \pi$, by Summary 7 any neighborhood of the double eigenvalue $\lambda_{n}\left(\varepsilon_{n}\right)$ contains a nonreal number from $\sigma(L(q))$. Thus, by Definition $1, \lambda_{n}\left(\varepsilon_{n}\right)$ is a complexation point. In the same way we prove that $\lambda_{n}\left(\pi-\delta_{n}\right)$ is a complexation points.

(d) By (13) and Theorem 1 the subset $\left\{\lambda_{n}(t): t \in\left[\varepsilon_{n}, \pi-\delta_{n}\right]\right\}$ of $\Gamma_{n}$ is an interval of the real line. To prove the relation $\gamma\left(0, \varepsilon_{n}\right) \subset \mathbb{C} \backslash \mathbb{R}$, first let us prove that $\left\{\lambda_{n}(t): t \in\left(0, \varepsilon_{n}\right)\right\} \subset$ $\mathbb{C} \backslash \mathbb{R}$. Suppose there exists $t_{0} \in\left(0, \varepsilon_{n}\right)$ such that $\lambda_{n}\left(t_{0}\right)$ is a real number. Then by Theorem $1\left\{\lambda_{n}(t): t \in\left[t_{0}, \varepsilon_{n}\right)\right\} \subset \mathbb{R}$. It with the first statement of $(d)$ implies that there exists a small neighborhood $U:=\left\{\lambda \in \mathbb{C}:\left|\lambda-\lambda_{n}\left(\varepsilon_{n}\right)\right|<\delta\right\}$ of $\lambda_{n}\left(\varepsilon_{n}\right)$ which does not contain a nonreal number from $\Gamma_{n}$. If $\lambda_{-n}(t) \in U \backslash \mathbb{R}$ for some $t \in[0, h]$, then $\overline{\lambda_{-n}(t)} \neq \lambda_{-n}(t)$ and by Summary 6 both are eigenvalues of $L_{t}(q)$ satisfying (11). Then there exist three eigenvalues (counting the multiplicity) satisfying (11) which contradicts Summary 9. Hence $U$ does not contain a nonreal number from $\Gamma_{-n}$ too. Moreover, it readily follows from summaries 8 and 9 that $U \cap \Gamma_{k}=\varnothing$ for all $k \neq n,-n$. Thus $U$ does not contain a nonreal number from $\sigma(L(q))$. However it is impossible, since $\lambda_{n}\left(\varepsilon_{n}\right)$ is a complexation point. It remains to prove that $\lambda_{n}(0)$ is not a real number. If we suppose to the contrary that $\lambda_{n}(0)$ is a real number, then we obtain that the curve $\left\{\lambda_{n}(t): t \in\left[0, \varepsilon_{n}\right]\right\}$ with its symmetry forms a closed curve in the spectrum, which contradicts Summary 5. Thus $\gamma\left(0, \varepsilon_{n}\right) \subset \mathbb{C} \backslash \mathbb{R}$. The proof of $\gamma\left(\pi, \delta_{n}\right) \subset \mathbb{C} \backslash \mathbb{R}$ is the same

Definition 2 If $n>N(h)$, then the pure nonreal parts $\gamma\left(0, \varepsilon_{n}\right)$ and $\gamma\left(\pi, \delta_{n}\right)$ of $\Gamma_{n}$ are said to be the left and right tails of $\Gamma_{n}$, where $\gamma\left(0, \varepsilon_{n}\right)$ and $\gamma\left(\pi, \delta_{n}\right)$ are defined in Theorem 4 . If $n<-N(h)$ then we interchange the words left and right.

Theorem 5 Suppose that (2) holds and $n>N(h)$. Then the band $\Gamma_{n}$ has left (right) nonreal tail and contains a left (right) complexation point $\lambda_{n}\left(\varepsilon_{n}\right)\left(\lambda_{n}\left(\pi-\delta_{n}\right)\right)$, where $\varepsilon_{n} \in(0, h]$ $\left(\left(\pi-\delta_{n}\right) \in[\pi-h, \pi)\right.$, if and only if $\lambda_{n}(0)\left(\lambda_{n}(\pi)\right)$ is a nonreal number. The theorem continuous to hold if the condition $n>N(h)$ and the word left (right) are replaced by $n<-N(h)$ and the word right (left). 
Proof. We prove the theorem for $n>N(h)$ and $\lambda_{n}(0)$. The proofs for the other cases are the same. If the eigenvalue $\lambda_{n}(0)$ is a real number then by Theorem $1\left\{\lambda_{n}(t): t \in[0, h]\right\} \subset \mathbb{R}$, since $\lambda_{n}(h)$ is also real number (see Theorem $3(a)$ ). It means that the left tail absent and by Theorem $4(d)$ the left complexation point absent. Now suppose that $\lambda_{n}(0)$ is a nonreal number. Denote by $t_{0}$ the smallest number in $[0, h]$ such that $\lambda_{n}\left(t_{0}\right)$ is real. Since $\lambda_{n}(0)$ is nonreal number, $\lambda_{n}(h)$ is real number and $\lambda_{n}$ is a continuous function, we have $t_{0} \in(0, h]$. Then $\left\{\lambda_{n}(t): t \in\left[t_{0}, \pi-h\right]\right\} \subset \mathbb{R}$ and any neighborhood of $\lambda_{n}\left(t_{0}\right)$ contains a nonreal number $\lambda_{n}(t)$, where $t \in\left[0, t_{0}\right)$. It means that $\lambda_{n}\left(t_{0}\right)$ is a complexation point and $\left\{\lambda_{n}(t): t \in\left[0, t_{0}\right)\right\}$ is the left tail of $\Gamma_{n}$

Now we find the necessary and sufficient conditions in which $\sigma(L(q))$ is a half line.

Theorem 6 Suppose that (2) holds. Then the spectrum of $L(q)$ is a half line if and only if the followings hold:

(i) one eigenvalue of $L_{0}(q)$ is simple and the all others are double,

(ii) all eigenvalues of $L_{\pi}(q)$ are double.

Proof. First suppose that $\sigma(L(q))=[a, \infty)$ for same $a$. Then the bands $\Gamma_{n}$ of the spectrum $\sigma(L(q))$ are the intervals of the real line and the left end of the first interval is $a$. Since these intervals may have at most one common points (see Proposition 1(b)), that is, do not overlap each other and $a$ is the leftmost point of the spectrum it is the end only of one band of the spectrum. Therefore by Remark 1 it is a simple eigenvalue. Moreover, using the asymptotic formulas (14) for $F(\lambda)$ and taking into account that $F(\lambda) \in \mathbb{R}$ for $\lambda \in \mathbb{R}$ (see Summary 7) we see that the graph $\{(\lambda, F(\lambda)): \lambda \in \mathbb{R})\}$ of $F(\lambda)$ intersect the line $y=2$ before the line $y=-2$. Therefore $a$ is the simple eigenvalue of $L_{0}(q)$. Thus by Proposition $1(b)$ the right end point $b$ of the first band is the eigenvalue of $L_{\pi}(q)$. Since $\sigma(L(q))$ has no gaps, $b$ is the left end point of the second interval. Therefore $b$ is the double eigenvalue of $L_{\pi}(q)$. The right end point of the second interval is the eigenvalue $c$ of $L_{0}(q)$. Since $\sigma(L(q))$ has no gaps, $c$ is the left end point of the third interval and hence $c$ is the double eigenvalue of $L_{0}(q)$. Continuing this procedure we obtain that all eigenvalues of $L_{0}(q)$ except $a$ and all eigenvalues of $L_{\pi}(q)$ are double.

Now suppose that $(i)$ and $(i i)$ holds. Let $\lambda_{k}(0)$ be the simple eigenvalue of $L_{0}(q)$. Then by Remark 1 it is end point of only one band $\Gamma_{k}$. If $\lambda_{k}(0)$ is nonreal then by Summary 6 , $\overline{\lambda_{k}(0)}$ is also an eigenvalue of $L_{0}(q)$ and is end point of only one band. It implies that $\overline{\lambda_{k}(0)}$ is also a simple eigenvalue of $L_{0}(q)$ which contradict to $(i)$. Thus $\lambda_{k}(0)$ is real.

Now consider the connectedness of $\sigma(L(q))$. Let $m>\max \{|k|, N(h)\}$. Then it readily follows from Proposition $2(b)$ and $(i),(i i)$ that

$$
\lambda_{m}(\pi)=\lambda_{-m-1}(\pi), \lambda_{-n}(0)=\lambda_{n}(0), \lambda_{n}(\pi)=\lambda_{-n-1}(\pi), \forall n>m .
$$

It means that $\Gamma:=\bigcup_{n:|n|>m} \Gamma_{n}$ is a connected curve. Consider $\gamma=\bigcup_{n:|n| \leq m} \Gamma_{n}$. Suppose that $\gamma$ is not a connected curve, that is, there exist at least two separated curves $\gamma_{1}$ and $\gamma_{2}$ lying in $\gamma$. Then $\gamma_{1}$ and $\gamma_{2}$ have 4 end points denoted by $a_{1}, a_{2}, a_{3}$ and $a_{4}$. One of them, say $a_{1}$ is $\lambda_{k}(0)$. It readily follows from Proposition $2(b)$ that one of others, say $a_{4}$, is $\lambda_{m}(\pi) \in \Gamma$ and $a_{i}$ for $i=2,3$ do not belong to $\Gamma$. It is also clear that there exist $p$ such that $a_{2}$ is the end point of $\Gamma_{p}$ and is not end point of $\Gamma_{s}$ for $s \neq p$. It, by Remark 1, implies that $a_{2}$ is a simple eigenvalue of either $L_{0}(q)$ or $L_{\pi}(q)$. It contradicts $(i)$ and $(i i)$, since $a_{2} \neq a_{1}$. Thus we proved that $\gamma$ is a connected curve with end points $a_{1}$ and $a_{4}=\lambda_{m}(\pi) \in \Gamma$. Therefore $\sigma(L(q))$ is a connected curve with one left end point $a_{1} \in \mathbb{R}$. On the other hand, by Theorem $3(a)$ we have $\lambda_{n}(h) \in \mathbb{R}$ for all $n>m$. Therefore repeating the proof of Theorem $1(a)$, we see that the part of $\sigma(L(q))$ from $a_{1} \in \mathbb{R}$ to $\lambda_{n}(h) \in \mathbb{R}$, where $n>m$, is the interval $\left[a_{1}, \lambda_{n}(h)\right]$. Letting $n$ tend to infinity we get $\sigma(L(q))=\left[a_{1}, \infty\right)$ 


\section{Reality and non-reality of $\Gamma_{n}$ for large $n$}

To consider the reality of $\Gamma_{n}$ for large $n$ we use the following theorem.

Theorem 7 Suppose that (2) holds and $|n|>N(h)$. Then the followings are equivalent

(a) Band $\Gamma_{n}$ is the real interval.

(b) The eigenvalues $\lambda_{n}(0)$ and $\lambda_{n}(\pi)$ are the real numbers.

(c) $\lambda_{n}(t)$ for $t \in(0, \pi)$ are simple eigenvalues of $L_{t}(q)$.

(d) The eigenvalues $\lambda_{n}(t)$ for $t \in(0, \pi)$ are not the complexation points.

Proof. By Proposition 1(a) and Theorem 1(b) the statements $(a)$ and $(b)$ are equivalent. It readily follows from Theorem 4 that $(c)$ and $(d)$ are equivalent. Theorem $4(c)$ and Theorem 5 imply that $(b)$ and $(d)$ are equivalent.

Now we find the conditions on the Fourier coefficients of the PT-symmetric potential $q$ for reality and non-reality of $\Gamma_{n}$ for large $n$. For this first of all we need to consider the Fourier coefficient of the potentials (2). In general the locally integrable periodic function has no Fourier decomposition. However we can consider the Fourier coefficients

$$
q_{n}:=\left(q, e^{i 2 \pi n x}\right):=\int_{0}^{1} q(x) e^{-i 2 \pi n x} d x
$$

for $n \in \mathbb{Z}$. It is clear and well-known that if $q$ is PT-symmerric, then using the substitution $t=-x$ one can get the equality

$$
\overline{q_{n}}=\int_{0}^{1} \overline{q(x)} e^{i 2 \pi n x} d x=\int_{0}^{1} \overline{q(-t)} e^{-i 2 \pi n t} d t=\int_{0}^{1} q(t) e^{-i 2 \pi n t} d t=q_{n}
$$

which means that $q_{n} \in \mathbb{R}$ for all $n \in \mathbb{Z}$.

First to find the necessary and sufficient conditions on the potential for reality and nonreality of $\Gamma_{n}$ we construct some class of periodic PT-symmetric function as follows. Let $S_{p}$ be the set of 1 periodic PT-symmetric functions $q \in W_{1}^{p}[0,1]$ such that

$$
q(1)=q(0), q^{\prime}(1)=q^{\prime}(0), \ldots, q^{(s-1)}(1)=q^{(s-1)}(0)
$$

for some $s \leq p$ and there exist positive constants $c_{1}, c_{2}, c_{3}$ and $N>0$ satisfying

$$
\left|q_{n}\right|>c_{1} n^{-s-1} \& c_{2}\left|q_{n}\right| \leq\left|q_{-n}\right| \leq c_{3}\left|q_{n}\right|, \forall n>N .
$$

In particular, $S_{0}$ is the set of 1 periodic PT-symmetric functions $q \in L_{1}[0,1]$ satisfying

$$
\left|q_{n}\right|>c_{1} n^{-1} \& c_{2}\left|q_{n}\right| \leq\left|q_{-n}\right| \leq c_{3}\left|q_{n}\right|, \forall n>N .
$$

Besides we use the following formulas obtained in [23] (see (40)-(44) of [23]). In [23] we proved that $\lambda_{n}(t)$ satisfies the equations

$$
\left(\lambda-(2 \pi n)^{2}-t^{2}-\frac{1}{2}\left(A(\lambda, t)+A^{\prime}(\lambda, t)\right)\right)^{2}=D(\lambda, t),
$$

where $D=(4 \pi n t)^{2}+q_{2 n} q_{-2 n}+8 \pi n t C+C^{2}+q_{2 n} B^{\prime}+q_{-2 n} B+B B^{\prime}, C=\frac{1}{2}\left(A-A^{\prime}\right)$,

$$
\begin{gathered}
A(\lambda, t)=\sum_{k=1}^{\infty} a_{k}(\lambda, t), A^{\prime}(\lambda, t)=\sum_{k=1}^{\infty} a_{k}^{\prime}(\lambda, t), \\
a_{k}(\lambda, t)=\sum_{n_{1}, n_{2}, \ldots, n_{k}} q_{-n_{1}-n_{2}-\ldots-n_{k}} \prod_{s=1}^{k} q_{n_{s}}\left(\lambda-\left(2 \pi\left(n-n_{1}-. .-n_{s}\right)+t\right)^{2}\right)^{-1},
\end{gathered}
$$




$$
a_{k}^{\prime}(\lambda, t)=\sum_{n_{1}, n_{2}, \ldots, n_{k}} q_{-n_{1}-n_{2}-\ldots-n_{k}} \prod_{s=1}^{k} q_{n_{s}}\left(\lambda-\left(2 \pi\left(n+n_{1}+. .+n_{s}\right)-t\right)^{2}\right)^{-1} .
$$

The functions $B$ and $B^{\prime}$ are obtained respectively from $A$ and $A^{\prime}$ by replacing $q_{-n_{1}-n_{2}-\ldots-n_{k}}$ with $q_{2 n-n_{1}-n_{2}-\ldots-n_{k}}$ and $q_{-2 n-n_{1}-n_{2}-\ldots-n_{k}}$. One can readily see from (20)-(22) that

$$
\left\{A(\lambda, t), A^{\prime}(\lambda, t), C(\lambda, t), B(\lambda, t), B^{\prime}(\lambda, t), D(\lambda, t)\right\} \in \mathbb{R}
$$

if $\lambda, t$ and the Fourier coefficients $q_{n}$ for $n \in \mathbb{Z}$ are real number. Moreover, there exists a constant $K$ such that the following inequalities hold (see (56) of [23])

$$
\left|A(\lambda, t)+A^{\prime}(\lambda, t)-A(\mu, t)-A^{\prime}(\mu, t)\right|<K n^{-2}|\lambda-\mu| .
$$

Besides the equalities

$$
C\left(\lambda_{n, j}(t), t\right)=t O\left(n^{-1}\right), B\left(\lambda_{n,}(t), t\right)=o\left(n^{-s-1}\right), B^{\prime}\left(\lambda_{n},(t), t\right)=o\left(n^{-s-1}\right)
$$

hold uniformly with respect to $t$ in $[0, h]$ (see (57) and (46) of [23]).

Remark 2 Formula (19) is proved in [23] under conditions (17) and (18) (see Theorem 2.4). In [25] we proved ([see Theorem 2 and (35), (37) of [25]]) that $\lambda_{n}(t)$ satisfies (19) without conditions (17) and (18) which readily follows from (37) and (38) of [23] (see the first paragraph of the proof of Theorem 2 of [25]).

Theorem 8 Suppose that (2) holds, $n$ is a large number and $t \in([0, h] \cup[\pi-h, \pi])$. Then the eigenvalue $\lambda_{n}(t)$ is real if and only if

$$
D\left(\lambda_{n}(t), t\right) \geq 0 .
$$

Proof. We prove the theorem $t \in[0, h]$. The proof for $t \in[\pi-h, \pi]$ is the same. Suppose that $\lambda_{n}(t)$ is real. Since the Fourier coefficients of the PT-symmetric function $q$ are real numbers (see (16)), by (23) we have

$$
F\left(\lambda_{n}(t), t\right):=\left(\lambda_{n}(t)-(2 \pi n)^{2}-t^{2}-1 / 2\left(A\left(\lambda_{n}(t), t\right)+A^{\prime}\left(\lambda_{n}(t), t\right)\right)\right) \in \mathbb{R} .
$$

Therefore the right side of (19) for $\lambda=\lambda_{n}(t)$ are nonnegative, that is, (26) holds.

Now suppose that (26) holds. Then, by (19), the relation (27) holds too. It remains to show that (27) implies the reality $\lambda_{n}(t)$. Suppose that $\lambda_{n}(t)$ is nonreal. Since $F\left(\lambda_{n}(t), t\right)$ is real number (see (27)), from (20)-(22) one can readily see that $F\left(\lambda_{n}(t), t\right)=F\left(\overline{\lambda_{n}(t)}, t\right)$ from which by using (24) we obtain the following contradiction

$$
\begin{aligned}
2\left|\lambda_{n}(t)-\overline{\lambda_{n}(t)}\right| & \left.=\mid A\left(\lambda_{n}(t), t\right)+A\left(\overline{\lambda_{n}(t)}, t\right)-A^{\prime}\left(\lambda_{n}(t), t\right)\right)-A^{\prime}\left(\overline{\lambda_{n}(t)}, t\right) \mid \\
& <2 K n^{-2}\left|\lambda_{n}(t)-\overline{\lambda_{n}(t)}\right| .
\end{aligned}
$$

The theorem is proved

The following corollary immediately follows from theorems 3,7 and 8 .

Corollary 1 Suppose that (2) holds and $n$ is a large number. Then $\Gamma_{n} \subset \mathbb{R}$ if and only if

$$
D\left(\lambda_{n}(0), 0\right) \geq 0 \quad \& \quad D\left(\lambda_{n}(\pi), \pi\right) \geq 0 .
$$

Now using this corollary, (18) and (25) we prove the following. 
Theorem 9 Suppose that $q \in S_{p}$ and $n$ is a large number. Then $\Gamma_{n} \subset \mathbb{R}$ if and only if

$$
q_{n} q_{-n}>0
$$

Proof. It follows from (25) that

$$
\begin{aligned}
& C\left(\lambda_{n}(0), 0\right)=0, D\left(\lambda_{n}(0), 0\right)=q_{2 n} q_{-2 n}+q_{2 n} B^{\prime}+q_{-2 n} B+B B^{\prime}, \\
& D\left(\lambda_{n}(0), 0\right)=q_{2 n} q_{-2 n}+o\left(q_{-2 n} n^{-s-1}\right)+o\left(q_{2 n} n^{-s-1}\right)+o\left(n^{-2 s-2}\right) .
\end{aligned}
$$

This with (18) implies that

$$
D\left(\lambda_{n}(0), 0\right)=q_{2 n} q_{-2 n}(1+o(1))
$$

as $n \rightarrow \infty$. Now suppose that (29) holds. Then by Theorem 2.12 of [23] the eigenvalue $\lambda_{n}(t)$ is simple for all $t \in[0, \pi]$. Therefore, by Theorem $7, \Gamma_{n}$ is real.

Now suppose that $\Gamma_{n}$ is real. Then by Corollary $1,(28)$ holds. The first equality in (28) with (31) imply that $q_{2 n} q_{-2 n}>0$, since $q_{2 n} q_{-2 n}$ is real (see (16)) and nonzero (see (18)). In the same way using the second equality in (28) we obtain $q_{2 n+1} q_{-2 n-1}>0$

In [18] we proved that if $q \in W_{1}^{s}[0,1]$ and (17) holds then

$$
\begin{gathered}
B\left(\lambda_{n}(0), 0\right)=-S_{2 n}+2 Q_{0} Q_{2 n}+o\left(n^{-s-2}\right), \\
B^{\prime}\left(\lambda_{n}(0), 0\right)=-S_{-2 n}+2 Q_{0} Q_{-2 n}+o\left(n^{-s-2}\right),
\end{gathered}
$$

where $Q_{k}$ and $S_{k}$ are the Fourier coefficients of the function $Q$ and $S$ defined by

$$
Q(x)=\int_{0}^{x} q(t) d t, \quad S(x)=Q^{2}(x)
$$

(see Lemma 6 of [18] ) and $-S_{ \pm 2 n}+2 Q_{0} Q_{ \pm 2 n}$ are real numbers (see page 655). Moreover

$$
q_{n}=o\left(n^{-s}\right), S_{ \pm 2 n}=o\left(n^{-s-1}\right), Q_{ \pm 2 n}=o\left(n^{-s-1}\right)
$$

(see page 658 of [18] ). Therefore using (30), (32) and (33) we obtain

$$
D\left(\lambda_{n}(0), 0\right)=P_{2 n}+o\left(n^{-2 s-2}\right),
$$

where $P_{n}=q_{n} q_{-n}-q_{n}\left(S_{-n}-2 Q_{0} Q_{-n}\right)-q_{-n}\left(S_{n}-2 Q_{0} Q_{n}\right)$. Similarly

$$
D\left(\lambda_{n}(\pi), \pi\right)=P_{2 n+1}+o\left(n^{-2 s-2}\right),
$$

If there exist $c$ such that

$$
\left|P_{n}\right|>c n^{-2 s-2}
$$

then we have

$$
D\left(\lambda_{n}(0), 0\right)=P_{2 n}(1+o(1)), D\left(\lambda_{n}(\pi), \pi\right)=P_{2 n+1}(1+o(1)) .
$$

Therefore using (35), Theorem 8 and Theorem 5 and taking into account that if $q \in W_{1}^{s}[0,1]$ and (2) holds then (17) holds too we obtain

Theorem 10 If $q \in W_{1}^{s}[0,1]$, (2) and (34) hold, $n$ is a large number and $P_{n}<0$ then $\Gamma_{n}$ has the nonreal tails. 
Now let us consider the connections between the reality of $\sigma(L(q))$ and the spectrality of $L(q)$ for $q \in S_{p}$. In [13] it was proved that $L(q)$ is a spectral operator if and only if

$$
\sup _{\gamma \in R}\left(\sup _{t \in(-\pi, \pi]}\|e(t, \gamma)\|\right)<\infty
$$

where $e(t, \gamma)$ be the spectral projection defined by contour integration of the resolvent of $L_{t}(q), \gamma \in R$ and $R$ is the ring consisting of all sets which are the finite union of the half closed rectangles (see Theorem 3.5). Note that the spectral singularities of the operator $L(q)$ are the points of its spectrum in neighborhoods of which the projections of $e(t, \gamma)$ are not uniformly bounded (see [9] and [21-23]). Therefore if $L(q)$ has a spectral singularity then it is not spectral operator. However may be $\sigma(L(q))$ does not contain a spectral singularity nevertheless $L(q)$ is not a spectral operator. It happens if $\|e(t, \gamma)\| \rightarrow \infty$ as $\gamma$ goes to infinity. In this case we say that $L(q)$ has a spectral singularity at infinity (see Definition 3.2 in [23]). According to (36), we say that $L(q)$ as an asymptotically spectral operator if the inequality obtained from (36) by replacing $R$ with $R(C)$ holds, where $C$ is a large positive number and $R(C)$ is the ring consisting of all sets which are the finite union of the half closed rectangles lying in $\{\lambda \in \mathbb{C}:|\lambda|>C\}$ (see Definition 3.6 in [23]). If $L(q)$ is an asymptotically spectral operator then it has noncomplicated spectral expansion (see Theorem 4 in [27]).

Theorem 11 (a) Let $|n|>N(h)$ and $a \in(0, \pi)$. Then the followings are equivalent.

1) $\lambda_{n}(a)$ is a spectral singularity of $L(q)$.

2) $\lambda_{n}(a)$ is a complexation point of $\sigma(L(q))$.

3) $\lambda_{n}(a)$ is a multiple eigenvalue of $L_{a}(q)$.

4) If $a \in(0, h]$ then $\left\{\lambda_{n}(t): t \in[0, a)\right\}$; if $a \in[\pi-h, \pi)$ then $\left\{\lambda_{n}(t): t \in(a, \pi]\right\}$ is a nonreal tail of $\Gamma_{n}$.

(b) Let $q \in S_{p}$ for some $p=0,1, \ldots$ Then the followings are equivalent.

1) $L(q)$ is an asymptotically spectral operator.

2) There exists $m \geq N(h)$ such that (29) holds for all $|n|>m$.

3) There exist $m \geq N(h)$ such that $\Gamma_{n}$ for $|n|>m$ are real pairwise disjoint intervals separated by the gaps of the spectrum.

Proof. (a) By Theorem 4, 2), 3) and 4) are equivalent. On the other hand, by Proposition 2 of [26] $\lambda_{n}(a)$ is a spectral singularity if and only if it a multiple eigenvalue.

(b) First let us show that 2) and 3) are equivalent. By Theorem 9, 3) implies 2). Now suppose that 2) holds. Then again by Theorem $9, \Gamma_{n}$ are real for all $|n|>m$. On the other hand if (29) holds then by Theorem 2.12 of [23] the eigenvalues $\lambda_{n}(t)$ for $t \in[0, \pi]$ are simple and hence by by Remark $1, \Gamma_{n}$ for $|n|>m$ are real pairwise disjoint intervals, that is 3 ) holds. In [23] we proved that if (29) holds then $L(q)$ is asymptotically spectral operator, that is, if 2) holds then 1) holds too. Now suppose that 2) does not hold. Then by Theorem 9 there exists a sequence $\left\{n_{k}\right\}$ such that $\Gamma_{n_{k}}$ is not a real interval that is contains a nonreal tail. Then by $(a)$ there exist $t_{n_{k}} \in(0, \pi)$ such that $\lambda_{n_{k}}\left(t_{n_{k}}\right)$ is a spectral singularity. It means that the conditions of Theorem 1(c) of [27] does not hold and hence $L(q)$ is not an asymptotically spectral operator. Thus 2) and 1) are equivalent

Now we consider a simple example that helps to see the complexity of the relations between the spectrum and spectrality of the PT-symmetric periodic operators.

Example 1 The operators $L_{t}(q)$ and $L(q)$ are denoted by $H_{t}(a, b)$ and $H(a, b)$ when

$$
q(x)=a e^{-i 2 \pi x}+b e^{i 2 \pi x},
$$

where $a$ and $b$ are the real numbers, that is, $q$ is a PT-symmetric potential. 
To consider the spectrum of these operators we use the following results of [24] formulated as Summary 10 (see Theorem 1 and (26) of [24]).

Summary 10 If $a b=c d$, then $\sigma(H(a, b))=\sigma(H(c, d))$ and $\sigma\left(H_{t}(a, b)\right)=\sigma\left(H_{t}(c, d)\right)$. The operators $\left.H_{t}(a, b)\right)$ and $H_{t}(c, d)$ have the same characteristic equation (4).

By this summary if $a b>0$ then the spectra of $H(c, d)$ and $H_{t}(a, b)$ coincide with the spectrum of the self adjoint operators $L(2 c \cos 2 \pi x)$ and $L_{t}(2 c \cos 2 \pi x)$ respectively, where $c$ is a positive square root of $a b$. It is well-known that (see Chapter 21 of [19] and Chapter 2 of [7]) all eigenvalues of $L_{t}(2 c \cos 2 \pi x)$ for all $t \in[0,2 \pi)$ are real and simple and all gaps in the spectrum of $L(2 c \cos 2 \pi x)$ are open. Therefore we have the following.

Theorem 12 If $a b>0$ then all eigenvalues of $H_{t}(a, b)$ for all $t \in[0,2 \pi)$ are real and simple and the spectrum of $H(a, b)$ consist of the real intervals $\Gamma_{n}$ separated by gaps, where $H_{t}(a, b)$ and $H(a, b)$ are defined in Example 1.

To consider the spectrality of $H(a, b)$ we use the following results of [25] and [27] formulated as Summary 11 (see Prop. 3 and Theorem 6 of [25] and Corollary 1 of [27]).

Summary 11 (a) If $|a| \neq|b|$, then the operator $H(a, b)$ has the spectral singularity at infinity and hence is not an asymptotically spectral operator.

(b) The operator $H(a, b)$ is an asymptotically spectral operator and has no spectral singularity at infinity if and only if

$$
|a|=|b|, \inf _{q, p \in \mathbb{N}}\{|q \alpha-(2 p-1)|\} \neq 0,
$$

where $\alpha=\pi^{-1} \arg (a b)$.

This summary immediately yields the following.

Theorem 13 The PT-symmetric operator $H(a, b)$ is a spectral operator if and only if $a=b$, that is, (37) is the real potential $2 a \cos 2 \pi x$

Proof. If $a=b$ then the operator $H(a, b)$ is the self-adjoint operator $L(2 a \cos 2 \pi x)$ and hence is the spectral operator. Now suppose that $a \neq b$. Since $a$ and $b$ are the real numbers, it is possible in the following cases. Case 1: $|a| \neq|b|$ and Case 2: $b=-a$. In Case 1 by Summary $11(a)$, the operator $H(a, b)$ has the spectral singularity at infinity and hence is not a spectral operator. In Case 2 we have $\pi^{-1} \arg (a b)=1$. Therefore, by Summary 11(b), $H(a, b)$ is not a spectral operator

Now using Theorem 4 and Theorem 5 we prove the following.

Theorem 14 Suppose that (2) holds. If there exists $m>N(h)$ such that $\lambda_{n}(0)$ and $\lambda_{n}(\pi)$ for $n>m$ are nonreal numbers then there exists $R$ such that $[R, \infty) \subset \sigma(L(q))$ and the number of gaps in the real part $\operatorname{Re}(\sigma(L(q)))$ of $\sigma(L(q))$ is finite.

Proof. Let and $n>m+1>N(h)+1$. By Theorem 5 there exist complexation points $\lambda_{n}\left(\varepsilon_{n}\right)$ and $\lambda_{n}\left(\pi-\delta_{n}\right)$, where $\varepsilon_{n} \in(0, h],\left(\pi-\delta_{n}\right) \in[\pi-h, \pi)$. Then using Theorem $4(d)$ and taking into account that $\Gamma_{n}$ is a simple open curve (see Proposition 1(b)) and $\lambda_{n}\left(\varepsilon_{n}\right)<\lambda_{n}\left(\pi-\delta_{n}\right)$ (see (11) and (12)) we obtain

$$
\operatorname{Re}\left(\Gamma_{n}\right)=\left\{\lambda_{n}(t): t \in\left[\varepsilon_{n}, \pi-\delta_{n}\right]\right\}=\left[\lambda_{n}\left(\varepsilon_{n}\right), \lambda_{n}\left(\pi-\delta_{n}\right)\right] .
$$

It follows from Summary 6 and Summary 9 that $\lambda_{-n}(0)$ is also nonreal number. Therefore in the same way and using (13) we prove that the right end point of $\operatorname{Re}\left(\Gamma_{-n}\right)$ is $\lambda_{-n}\left(\varepsilon_{n}\right)$ 
and coincides with the left ent point of $\operatorname{Re}\left(\Gamma_{n}\right)$. Instead of $\lambda_{n}(0)$ using $\lambda_{n}(\pi)$ and repeating this proof we obtain that the right end point of $\operatorname{Re}\left(\Gamma_{n}\right)$ coincides with the left ent point of $\operatorname{Re}\left(\Gamma_{-n-1}\right)$. Therefore there exist $R$ such that $[R, \infty) \subset \sigma(L(q))$. To complete the proof it is enough to note that by Theorem $1(a), \operatorname{Re}\left(\Gamma_{n}\right)$ for each $n \in \mathbb{Z}$ is either empty set or a point or an interval.

Now we find the conditions on the Fourier coefficients

$$
f_{n}=\int_{0}^{1} f(x) \cos 2 \pi n x d x, g_{n}=\int_{0}^{1} g(x) \sin 2 \pi n x d x
$$

for which the number of gaps in the real part of the spectrum of $L(q)$ is finite, where $f=\operatorname{Re} q$ and $g=\operatorname{Im} q$. It is clear that if $q$ is PT-symmerric, then $f$ and $g$ are even and odd functions respectively and hence

$$
q_{n}=f_{n}+g_{n}, q_{-n}=f_{n}-g_{n} .
$$

Using (34), (35), Theorem 8 and Theorem 14 and taking into account that if $q \in W_{1}^{s}[0,1]$ and (2) holds then (17) holds too we obtain we obtain.

Theorem 15 Suppose that (2) holds and $q \in W_{1}^{s}[0,1]$ for some $s \geq 0$. If there exist positive constants $m$ and $\alpha$ such that for $n>m$ the inequality

$$
P_{n}<-\alpha n^{-2 s-2}
$$

holds, then the number of gaps in the real part of the spectrum of $L(q)$ is finite.

Now we prove the other and more applicable theorem.

Theorem 16 Suppose that (2) holds and $q \in W_{1}^{s}[0,1]$ for some $s \geq 0$. If there exist $\delta>1$, $\beta>0$ and $m>0$ such that

$$
\left|g_{n}\right|>\beta n^{-s-1},\left|g_{n}\right|>\delta\left|f_{n}\right|
$$

for all $n>m$, then the number of gaps in the real part of the spectrum of $L(q)$ is finite, where $f_{n}$ and $g_{n}$ are the Fourier coefficients of $\operatorname{Re} q$ and $\operatorname{Im} q$ defined in (38).

Proof. It readily follows from (39) and (41) that

$$
\left|q_{n}\right|>\varepsilon n^{-s-1}, \quad\left|q_{-n}\right|>\varepsilon n^{-s-1}
$$

and hence

$$
-q_{n} q_{-n}=\left(g_{n}^{2}-f_{n}^{2}\right)>\gamma n^{-2 s-2}, \frac{\left|q_{ \pm n}\right|}{\left|q_{n} q_{-n}\right|}=O\left(n^{s+1}\right)
$$

for some $\varepsilon>0$ and $\gamma>0$. Using (2) and taking into account that $q^{(s)}$ is integrable on $[0,1]$ one can readily verify that the functions $Q$ and $S$ are 1 periodic functions, $Q^{(s+1)}$ and $S^{(s+1)}$ are integrable on $[0,1]$ and hence $S_{ \pm n}=o\left(n^{-s-1}\right)$ and $Q_{ \pm n}=o\left(n^{-s-1}\right)$. Therefore, using (42) and the definition of $P_{n}$ we obtain

$$
P_{n}=q_{n} q_{-n}-q_{n}\left(S_{-n}-2 Q_{0} Q_{-n}\right)-q_{-n}\left(S_{n}-2 Q_{0} Q_{n}\right)=\left(f_{n}^{2}-g_{n}^{2}\right)(1+o(1)) .
$$

Thus if (41) holds then (40) holds too. Hence the proof follows from Theorem 15

Theorem 16 shows that one can construct the large and easily checkable classes of the finite-zone PT-symmetric periodic potentials, by constructing the potentials $q$ so that the Fourier coefficients of $g=\operatorname{Im} q$ is greater (by absolute value) than the Fourier coefficient of $f=\operatorname{Re} q$. One of them are given in the next theorem. 
Theorem 17 Suppose that (2) holds, $g \in W_{1}^{s+1}(a, a+1) \cap W_{1}^{s}[0,1]$ for some $s \geq 0$ and $a \in$ $[0,1)$ and $g^{(s)}$ has a jump discontinuity at a with size of the jump $c:=g^{(s)}(a+0)-g^{(s)}(a-0)$, while either $f \in W_{1}^{s+1}[0,1]$ or $f \in W_{1}^{s+1}(b, b+1) \cap W_{1}^{s}[0,1]$ for some $b \in[0,1)$ and $f^{(s)}$ has a jump discontinuity at $b$ with size of the jump $d:=f^{(s)}(b+0)-f^{(s)}(b-0)$. If $|d|<|c|$ then the number of gaps in the real part of the spectrum of $L(q)$ is finite.

Proof. The Fourier coefficient $g_{n}$ defined in (38) can be calculated by

$$
g_{n}=\lim _{\varepsilon \rightarrow 0} \int_{a+\varepsilon}^{a+1-\varepsilon} g(x) \sin 2 \pi n x d x .
$$

Applying $s+1$ times the integration by parts formula we get

$$
\left|g_{n}\right|=|c|(2 \pi n)^{-s-1}+o\left(n^{-s-1}\right) .
$$

In the same way we obtain that either $f_{n}=o\left(n^{-s-1}\right)$ or

$$
\left|f_{n}\right|=|d|(2 \pi n)^{-s-1}+o\left(n^{-s-1}\right) .
$$

Therefore if $|d|<|c|$, then (41) holds and hence the proof follows from Theorem 16

Conclusion 1 Theorem $4(d)$ shows that the main part of the spectrum of $L(q)$ with PTsymmetric periodic potential is real. Indeed, since $\varepsilon_{n} \rightarrow 0$ and $\delta_{n} \rightarrow 0$ as $n \rightarrow \infty$, the length of the real part $\left\{\lambda_{n}(t): t \in\left[\varepsilon_{n}, \pi-\delta_{n}\right]\right\}$ of $\Gamma_{n}$ for $n>N(h)$ is of order $4 \pi^{2} n$, while the length of the nonreal tails $\left\{\lambda_{n}(t): t \in\left[0, \varepsilon_{n}\right)\right\}$ and $\left\{\lambda_{n}(t): t \in\left(\pi-\delta_{n}, \pi\right]\right\}$ is $O\left(n \varepsilon_{n}\right)$ and $O\left(n \delta_{n}\right)$ respectively. Moreover, instead of (14) using more sharp estimation one can show that $\varepsilon_{n}$ and $\delta_{n}$ approach zero rapidly for smooth potential. For example if $q \in S_{p}$ then $q_{n}=o\left(n^{-s}\right)$ and the points $\varepsilon_{n}, \pi-\delta_{n}$ corresponds to zero of $D$ defined in (19). It with (25) implies that $\varepsilon_{n}=o\left(n^{-s-1}\right), \delta_{n}=o\left(n^{-s-1}\right)$. Therefore the length of the nonreal tails of $\Gamma_{n}$ is o $\left(n^{-s}\right)$. Thus the spectrum of the PT-symmetric Hill operator is asymptotically real and the spectrum of the self-adjoint Hill operator is completely real. Therefore one can say that they have a closely spectral characteristic.

However, besides the reality of the spectrum the self-adjoint operators have the following main characteristics: they have orthogonal projections, spectral resolution of the identity operator, spectral representation and have no spectral singularities. The reality of spectrum of PT-symmetric operator does not guarantee to hold the listed characteristics of the selfadjoint operator for the PT-symmetric operator. For example, if

$$
q(x+1)=q(x), q \in L_{1}[0,1], q_{-n}=0, q_{n} \in \mathbb{R} \backslash\{0\}, \forall n=1,2, \ldots
$$

then $q$ is PT-symmetric potential and the spectrum is completely real: $\sigma(L(q))=[0, \infty)$. Nevertheless $L(q)$, in general, has no orthogonal projections and has infinitely many spectral singularities (see [8, 26]). In this example $\lambda_{n}(0)$ and $\lambda_{n}(\pi)$ are the spectral singularities if they are double eigenvalues and there exist associated function corresponding to their eigenfunctions. Moreover, it happens for almost all potentials (43) (see [28]). If $\lambda_{n}(0)$ and $\lambda_{n}(\pi)$ are simple and nonreal for $n>N(h)$ then $\Gamma_{n}$ has complexation points $\lambda_{n}\left(\varepsilon_{n}\right)$ and $\lambda_{n}\left(\pi-\delta_{n}\right)$ which are spectral singularities (see Theorem 11(a)). Thus, in this case $L(q)$ also has infinitely many spectral singularities and hence is not asymptotically spectral operator.

Theorem 11 shows that if $\lambda_{n}(0)$ and $\lambda_{n}(\pi)$ are real and simple eigenvalues for large $n$ then the PT-symmetric operator $L(q)$ may have only finitely many spectral singularities and is asymptotically spectral. Hence the simplicity plus reality of $\lambda_{n}(0)$ and $\lambda_{n}(\pi)$ for large $n$, namely the condition (29) on the potential $q$, provide the PT-symmetric operator $L(q)$ to be close to the self adjoint Hill operator $L(q)$ for $q \in S_{p}$. 
Finally, note that Theorem 11(b) (the equivalence of 1) and 3)) shows the asymptotic connection of reality of $\sigma(L(q))$ and spectrality of $L(q)$ for $q \in S_{p}$. On the other hand, theorems 12 and 13 for Example 1 show that if $a b>0$ and $a \neq b$ then the spectrum of $H(a, b)$ coincides with the spectrum of the self-adjoint Mathieu-Hill operator, $L(2 c \cos 2 \pi x)$, all eigenvalues of $H_{t}(a, b)$ for all $t \in[0,2 \pi)$ are real and simple and hence $\sigma(H(a b))$ has no spectral singularities and complexation points, while $H(a, b)$ is not a spectral operator. Thus the relation between the spectrum and spectrality of the PT-symmetric periodic operators is complicated and depends on the subclasses of the PT-symmetric potentials.

\section{References}

[1] Z. Ahmed, Energy band structure due to a complex, periodic, PT -invariant potential Phys. Lett.A, 286 (2001), 231-235.

[2] Non-Selfadjoint Operators in Quantum Physics: Mathematical Aspects, First Edition. Edited by Fabio Bagarello, Jean-Pierre Gazeau, Franciszek Hugon Szafraniec and Miloslav Znojil, John Wiley \& Sons, Inc. Published, 2015.

[3] C. M. Bender, G. V. Dunne and P. N. Meisinger, Complex periodic potentials with real band spectra Phys. Lett. A, 252 (1999), 272-276.

[4] E Caliceti, S Graffi, Reality and non-reality of the spectrum of PT -symmetric operators: Operator-theoretic criteria, Pramana Journal of Physics, 73 (2009), 241-249

[5] J. M. Cervero, PT -symmetry in one-dimensional quantum periodic potentials Phys. Lett. A, 317 (2003), 26-31.

[6] J. M. Cervero and A. Rodriguez, The band spectrum of periodic potentials with PT -symmetry J. Phys. A:Math. Gen. 37 (2004), 10167-10177.

[7] M. S. P. Eastham, The Spectral Theory of Periodic Differential Operators, New York: Hafner, 1974.

[8] M. G. Gasymov, Spectral analysis of a class of second-order nonself-adjoint differential operators, Fankts. Anal. Prilozhen 14 (1980), 14-19.

[9] F. Gesztesy and V. Tkachenko, A criterion for Hill's operators to be spectral operators of scalar type, J. Analyse Math., 107 (2009), 287-353.

[10] H. F. Jones, The energy spectrum of complex periodic potentials of Kronig-Penney type Phys. Lett. A, 262 (1999), 242-244.

[11] K.G. Makris, R. El-Ganainy, D.N. Christodoulides, Z.H. Musslimani, PT-Symmetric Periodic Optical Potentials, Int J Theor Phys, 50 (2011), 1019-1041.

[12] V. A. Marchenko, "Sturm-Liouville Operators and Applications," Birkhauser Verlag, Basel, 1986.

[13] D. C. McGarvey, Differential operators with periodic coefficients in $L_{p}(-\infty, \infty)$, J. Math. Anal. Appl. 11 (1965), 564-596.

[14] D. C. McGarvey, Perturbation results for periodic differential operators in $L_{p}(-\infty, \infty)$, J. Math. Anal. Appl. 12 (1965), 187-234.

[15] A. Mostafazadeh, Psevdo-hermitian representation of quantum mechanics, International Journal of Geometric Methods in Modern Physics, 11 (2010),1191-1306. 
[16] F. S. Rofe-Beketov, The spectrum of nonselfadjoint differential operators with periodic coefficients, Soviet Math. Dokl. 4 (1963), 1563-1566.

[17] K. C. Shin, On the shape of spectra for non-self-adjoint periodic Schrödinger operators, Journal of Physics A: Mathematical and General, 37 (2004), 8287-8291.

[18] A. A. Shkalikov, O. A. Veliev, On the Riesz basis property of the eigen- and associated functions of periodic and antiperiodic Sturm-Liouville problems, Math. Notes, 85 (2009), 647-660.

[19] E. C. Titchmarsh, "Eigenfunction Expansions Associated with Second Order Differential Equations," Part II, Oxford Univ. Press, London, 1958.

[20] V. A. Tkachenko, Spectral analysis of nonselfadjoint Schrodinger operator with a periodic complex potential, Soviet Math. Dokl., 5 (1964), 413-415.

[21] O. A. Veliev, The spectrum and spectral singularities of differential operators with complex-valued periodic coefficients. Differential Cprime Nye Uravneniya, 19 (1983), 1316-1324.

[22] O. A. Veliev, M. Toppamuk Duman, The spectral expansion for a nonself-adjoint Hill operators with a locally integrable potential, J. Math. Anal. Appl. 265 (2002), 76-90.

[23] O. A. Veliev, Asymptotic analysis of non-self-adjoint Hill's operators, Central European Journal of Mathematics, 11 (2013), 2234-2256.

[24] O. A. Veliev, Isospectral Mathieu-Hill operators, Letters in Mathematical Physics, 103 (2013), 919-925.

[25] O. A. Veliev, Spectral Analysis of the Non-self-adjoint Mathieu-Hill Operator, arXiv:1202.4735v4, (2013).

[26] O. A. Veliev, Spectral Problems of a Class of Non-self-adjoint One-dimensional Schrodinger Operators, J. Math. Anal. Appl., 422 (2015), 1390-1401.

[27] O. A. Veliev, On the spectral singularities and spectrality of the Hill's Operator, Operators and Matrices, 10 (2016), 57-71.

[28] O. A. Veliev, On a Class of Non-self-adjoint Multidimensional Periodic Schrodinger Operators, arXiv:1604.02061 v2 (2016). 